\title{
IVF in IBD: Initial Validation For Success?
}

\author{
Patricia Kozuch $^{1}$
}

Published online: 24 June 2016

(C) Springer Science+Business Media New York 2016

The primary clinical manifestation of inflammatory bowel disease (IBD) is idiopathic inflammation of the gastrointestinal-colonic mucosa. Given that IBD strikes most frequently in the teens to thirties [1], corresponding to the prime childbearing years for women, fertility is understandably a topic germane to this population. Whereas fertility rates for women medically managed for ulcerative colitis (UC) do not differ from those without UC, a threefold increase in infertility (average of 15-48 \%) has been reported in a meta-analysis of studies of women who have undergone total proctocolectomy with ileal pouchanal anastomosis (IPAA), believed to be secondary to postoperative adnexal adhesions [2]. Elevated post-operative infertility rates may be normalized following laparoscopically performed IPAA [3]. In Crohn's disease (CD), data are more equivocal regarding higher rates of infertility: voluntary childlessness (for a wide variety of reasons) may account for reported higher rates of infertility in past studies [4], although decreased ovarian reserve as measured by anti-Müllerian hormone occurs in $\mathrm{CD}$, particularly at age $>30$ and with colonic location of disease [5, 6]. Regardless of whether a woman with IBD has difficulty becoming pregnant related to surgery, disease or for other reasons, knowledge of what influences the success of in vitro fertilization (IVF) in this population as it compares to women without IBD is certainly welcome.

In this issue of Digestive Diseases and Sciences, Oza et al. [7] report their findings of a matched retrospective cohort study regarding specific factors associated with the

Patricia Kozuch

patricia.kozuch@jefferson.edu

1 Division of Gastroenterology and Hepatology, Thomas Jefferson University Hospital, Philadelphia, PA, USA success of IVF in women with IBD, a subanalysis of data derived from a larger retrospective study comparing IVF success in women with IBD relative to the general population [8]. Since this same group previously published a subanalysis examining IVF in women with UC who had undergone IPAA [9], this subanalysis is actually the third in a series that compared women with IBD undergoing IVF to the general infertility population. The first study identified 121 female patients with IBD (UC: $n=71$; CD: $n=49$; unclassified: $n=1$ ) from two tertiary IBD centers in Boston and 470 matched controls without IBD, all of whom underwent IVF between 1998 and 2011 at two affiliated centers for infertility. The study reported no differences in the cumulative rate of live births or incidence of pregnancy/live birth after the first cycle of IVF for IBD patients of either subtype compared to controls [8]. The second study described comparable rates of IVF birth rates in women with UC who underwent IPAA $(n=22)$ versus those with UC who had not undergone IPAA $(n=49)$ versus matched controls from the general infertility population [9].

In the current study, the same group digs more deeply into their data to determine what specific factors may influence success of IVF in the IBD population [7]. In the general population of women undergoing IVF, advanced maternal age, higher body mass index (BMI), and elevated serum concentrations of follicle stimulating hormone on day 3 of the menstrual cycle (CD3 FSH) are all associated with decreased success rate of IVF. Not unexpectedly, this study showed that women with IBD are not immune to these issues, as younger age and lower BMI at IVF were associated with achieving a live birth for patients with UC and $\mathrm{CD}$, as was lower CD3 FSH for CD patients. UC patients achieving live birth also had a shorter duration of disease. For both UC and CD, surgical history and 
medications did not influence the success of live birth whereas past use of biologics in three UC patients was associated with a diminished rate of live births, although with such small numbers, no statistically significant conclusions can be drawn. In $\mathrm{CD}$, there was a trend for decreased success of live births among patients who had total proctocolectomy and IPAA. While the vast majority of all IBD patients in this study were in clinical remission (nearly $85 \%$ UC and $80 \% \mathrm{CD}$ ), interestingly, live births occurred in $82 \%$ of UC patients with a partial Mayo score (an assessment for UC activity) of 0 compared to only $50 \%$ for those with a higher score. Not surprisingly, among those not achieving live births, more IVF cycles and higher gonadotropin doses for both IBD subtypes and more frequent use of assisted hatching (a technique used to increase rate of embryo implantation) for UC were observed.

As pointed out by the authors, the small sample size and retrospective application of activity indices are the most significant limitations of the study. That being said, having some notion of disease severity is an important factor to take into account given that pregnancy outcomes may be adversely affected when women enter pregnancy with active disease. The effect of medications, which may be a marker of disease severity and/or otherwise possibly affect IVF success, was also appropriately explored in this study, although many took no medications and very few received any immunosuppressive treatments. Patients included in this study received IBD care at a tertiary center, which may further affect the ability to generalize these results to patients cared for in the community. Of note, earlier this year, a large population-based Danish cohort study of IVF success in IBD (614 IBD patients vs. 52,489 women without IBD) related overall lower rates of live births for the IBD group, a more robust result in UC patients [10]. Interestingly, while no differences in the rate of live births for UC patients who had surgery were observed, a decreased rate of live births was reported in CD patients who had undergone surgery. While this study lacked data regarding disease severity, remission rates, and medication exposure, surgery in UC is considered curative, usually precluding the use of medications, whereas surgery in $\mathrm{CD}$ may be a marker for more severe disease. Neither smoking status nor comorbidities were noted in the current Oza study, and although these were not significant factors in the Danish cohort study, past smoking history was not noted. Given that UC patients are more likely to be ex-smokers and past smoking history may decrease success of IVF [11], current and past smoking status will be important patient characteristics to include in future analyses.
The current study provides initial data that is reassuring for IBD patients who are contemplating IVF. Women with IBD, just like those in the general population, should be counseled to maintain healthy weights and not postpone childbearing for too long given that obesity and advanced maternal age were both associated with decreased rates of live births. IVF also appeared to be most successful when mucosal healing was achieved. A larger prospective population-based study should ideally include data points such as past and present smoking exposure, the effect of IVF on IBD course, and postpartum disease history, data that should provide further insight into the factors affecting IVF success and pregnancy in IBD.

\section{References}

1. Loftus CG, Loftus EV Jr, Harmsen WS, et al. Update on the incidence and prevalence of Crohn's disease and ulcerative colitis in Olmsted County, Minnesota, 1940-2000. Inflamm Bowel Dis. 2007; 13:254-261.

2. Waljee A, Waljee J, Morris AM, et al. Threefold increased risk of infertility: a meta-analysis of infertility after ileal pouch anal anastomosis in ulcerative colitis. Gut. 2006;55:1575-1580.

3. Bartels SA, D'Hoore A, Cuesta MA, et al. Signficantly increased pregnancy rates after laparoscopic restorative proctocolectomy: a cross-sectional study. Ann Surg. 2012;256:1045-1048.

4. Tavernier N, Fumery M, Peyrin-Biroulet L, et al. Systematic review: fertility in non-surgically treated inflammatory bowel disease. Aliment Pharmacol Ther. 2013;38:847-853.

5. Senates E, Colak Y, Erdem ED, et al. Serum anti-Mullerian hormone levels are lower in reproductive-age women with Crohn's disease compared to healthy control women. J Crohns Colitis. 2013;7:29-34.

6. Freour T, Miossec C, Bach-Ngohou K, et al. Ovarian reserve in young women of reproductive age with Crohn's disease. Inflamm Bowel Dis. 2012;18:1515-1522.

7. Oza SS, Pabby V, Dodge LE et al. Factors associated with the success of in vitro fertilization in women with inflammatory bowel disease. Dig Dis Sci. (Epub ahead of print). doi:10.1007/ s10620-016-4076-7.

8. Oza SS, Pabby V, Dodge LE, et al. In vitro fertilization in women with inflammatory bowel disease is as successful as in women from the general infertility population. Clin Gastroenterol Hepatol. 2015;13:1641-1646.

9. Pabby V, Oza SS, Dodge LE, et al. In vitro fertilization is successful in women with ulcerative colitis and ileal pouch anal anastomosis. Am J Gastroenterol. 2015;110:792-797.

10. Norgard BM, Larsen PV, Fedder J, et al. Live birth and adverse birth outcomes in women with ulcerative colitis and Crohn's disease receiving assisted reproduction: a 20-year nationwide cohort study. Gut. 2016;65:767-776.

11. Klonoff-Cohen H, Natarajan L, Marrs R, et al. Effects of female and male smoking on success rates of IVF and gamete intraFallopian transfer. Hum Reprod. 2001;16:1382-1390. 